\title{
Dural metastasis masquerading as an en plaque meningioma
}

\author{
Ankur Kapoor, Amey Savardekar, Debajyaoti Chaterjee ${ }^{1}$, Pravin Salunke, Rakesh K Vasishta ${ }^{1}$, Anish Bhattacharya ${ }^{2}$ \\ Departments of Neurosurgery, ${ }^{1}$ Histopathology, and ${ }^{2}$ Nuclear medicine, Postgraduate Institute of Medical Education and Research (PGIMER), \\ Chandigarh, Haryana and Punjab, India
}

\section{ABSTRACT}

Metastatic adenocarcinoma mimicking meningioma is rare; and any metastatic lesion masquerading as an en plaque meningioma is extremely rare. We report the case of a 50-year-old female, who presented with headache and left hemiparesis for 1 month and on imaging showed a dural-based enhancing mass along the right hemisphere. The patient was operated with a working diagnosis of en plaque meningioma. Histopathology revealed metastatic adenocarcinoma. This report highlights an unusual radiological presentation of a metastatic lesion as dural based en plaque variety. Metastasis should be borne in mind for any en plaque lesion with rapid clinical progression.

Key words: Dural metastases, en plaque dural lesions, enplaque lesions, radiological mimic

\section{Introduction}

Clinical presentation with dural-based metastasis mimicking meningioma is very rare. Clinically and/or radiologically these lesions are often misinterpreted as meningioma, which may delay surgery and consequently have a deleterious impact on patient care. ${ }^{[1,2]}$ En plaque lesions refer to a flattened sheath of tumor, taking the shape of an underlying bone. We report a case of dural metastasis which on pre-operative imaging had the appearance of an en plaque meningioma. Histopathology showed metastatic adenocarcinoma. The primary lesion in lung was found on subsequent fluorodeoxyglucose positron emission tomography (FDG-PET) and chest CT scan.

\section{Case Report}

A 50-year-old female presented to us with headache, vision loss in the right eye and progressive left

\begin{tabular}{|l|l|}
\hline \multicolumn{2}{|c|}{ Access this article online } \\
\hline Quick Response Code: & Website: \\
\hline & www.ruralneuropractice.com \\
\cline { 2 - 2 } & \\
\hline
\end{tabular}

hemiparesis (grade 4/5) for 1 month. MRI revealed extra-axial dural-based enhancing mass all along the right hemispheric convexity dura with mass effect, simulating an en plaque meningioma [Figure 1]. The lesion was hypointense on T1WI, hyperintense on T2WI, and intensely enhancing on contrast administration. The atypical features were the disproportionate mass effect caused by the lesion, the highly irregular and nodular surface of the lesion and the presence of a heterogeneous interface between the lesion and the normal brain (suggestive of micro-bleeds). Also, on plain CT scan the bone overlying the lesion was found to be normal, without evidence of hyperostosis.

In view of rapid progression of symptoms, the patient underwent right fronto-parietal craniotomy and gross total excision of the lesion. The tumor was extra-axial though there were areas with poor arachnoid plane, suggesting invasion into brain parenchyma. Histopathology revealed metastatic adenocarcinoma. Whole body PET CT showed mass in the right lung apex with active lung nodules, mediastinal lymphadenopathy and metastasis in adjacent vertebral bodies [Figure 2]. The patient was started on adjuvant chemotherapy with irinotecan $100 \mathrm{mg}$ per day but went on to develop recurrence in 8 weeks time and expired at 4 months after surgery. The cause of death was progressive neurological deterioration due to intracranial tumor growth.

\section{Address for correspondence:}

Dr. Pravin Salunke, Postgraduate Institute of Medical Education and Research (PGIMER), Sector 12, Chandigarh - 160012 , Haryana and Punjab, India. E-mail: drpravin_salunke@yahoo.co.uk 


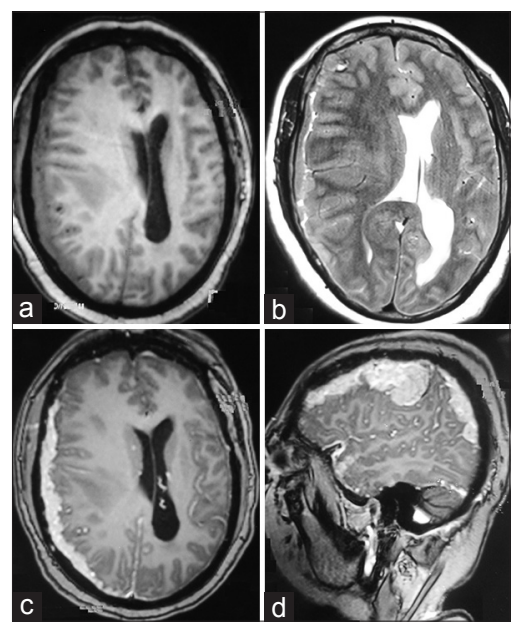

Figure 1: (a) Axial T1 image showing the dural-based lesion all along right convexity with mass effect. (b) T2 image showing the heterogeneous lesion with areas of hyperintensity. (c and d) T1 post-gadolinium axial and sagittal images showing contrast enhancement of the dural-based en plaque lesion

\section{Discussion}

Meningeal involvement as a presenting symptom of malignancy is rare. ${ }^{[3]}$ Primary neoplastic dural-based lesions are commonly meningiomas. Uncommon primary dural lesions include solitary fibrous tumors (SFT), hemangiopericytomas, sarcomas, chondromas, etc. ${ }^{[4]}$ Occasionally, non-neoplastic lesions like tuberculomas, neurosarcoidosis, plasma cell granulomas, Rosai Dorfman disease, xanthomas and rheumatoid nodule may present as a dural-based lesion. Primary brain tumors such as gliosarcoma may mimic a meningioma, particularly if associated with desmoplastic response from involved meninges. ${ }^{[1]}$ Of the dural-based metastatic lesions, carcinomas of breast, lung and prostate have been the most commonly reported. ${ }^{[4]}$

Typically, these tumors produce MRI images with increased signals on T2-weighted images and often with an enhancing dural tail mimicking a meningioma.${ }^{[4]} \mathrm{MR}$ spectroscopy of metastases has been found to have different lipid signals and lactate peaks to meningiomas..$^{[4]}$ However, radiological presentation of dural-based metastasis as en plaque variety is extremely rare. The clinical picture may help in such cases. Our patient presented with holocranial headache, right eye vision loss and progressive left hemiparesis. On retrospective evaluation after considering radiological and operative findings, the right eye vision loss was explained due to the involvement of the right optic nerve at the optic canal due to en plaque nature of the metastases. This was an unusual feature in the presentation. The left hemiparesis can easily be explained by the right hemispheric dural metastases. A rapid

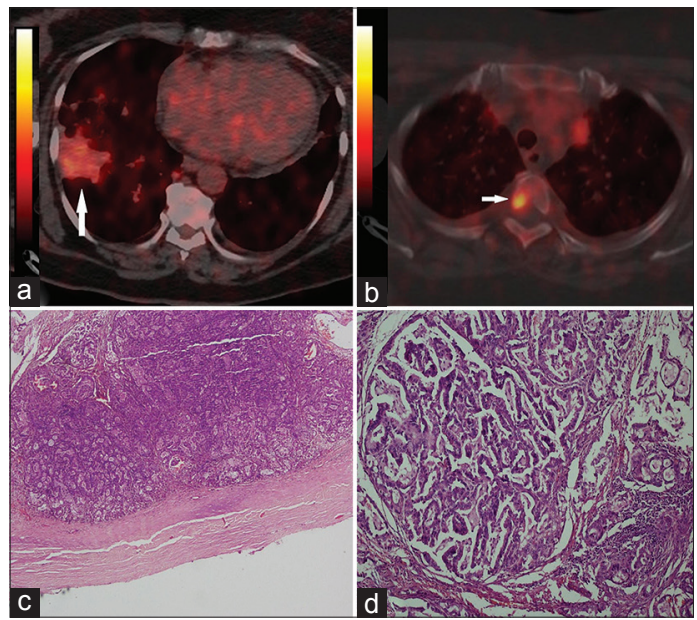

Figure 2: (a) PET scan showing FDG uptake in the right lung (arrow). (b) PET scan image showing uptake in vertebral body (arrow). (c) Photomicrograph showing a tumor attached to the inner surface of dura (HE, X2). (d) Tumor cells are arranged in a form of glands and trabeculae with mucin production (HE, X20)

progression of symptoms should have raised our suspicion that we were dealing with a malignant entity.

Dural involvement in metastatic lesions may be due to direct extension of metastatic skull metastases or by hematogenous spread through venous or arterial dissemination from the primary site. Histopathologic evaluation combined with a judicious immunohistochemical panel can reliably differentiate metastatic carcinomas from anaplastic meningioma, a known close differential diagnostic consideration. Histologic features usually reveal the metastatic nature of tumor. However, lobular growth may mimic whorls or a syncytial growth pattern, raising the possibility of a malignant meningioma. Because metastatic carcinomas usually exhibit more intense cytokeratin and no S-100 protein expression, immunohistochemical analysis generally excludes meningioma. ${ }^{[2]}$ Advances in chemotherapy may be altering the current incidence, but in the last decade, solitary metastasis to the dura in breast cancer occurred in approximately $8 \%$ of autopsied patients with this. ${ }^{[5]}$ By consensus, the prognosis of leptomeningeal carcinomatosis (LMC) is poor. The median survival in untreated patients is 4 to 6 weeks, which may increase to 4 to 6 months with aggressive treatment in some cases. ${ }^{[3]}$

The importance of awareness among oncologists, neurosurgeons, and neuropathologists that carcinomas of diverse primary sites can present with dural-based masses and radiologically mimicking meningiomas cannot be overstated. Metastasis should be kept in mind, even for a relatively benign looking en plaque lesion especially with rapid clinical progression. 


\section{References}

1. Laidlaw JD, Kumar A, Chan A. Dural metastases mimicking meningioma. Case report and review of the literature. J Clin Neurosci 2004;11:780-3.

2. Tagle P, Villanueva P, Torrealba G, Huete I. Intracranial metastasis or meningioma? An uncommon clinical diagnostic dilemma. Surg Neurol 2002;58:241-5.

3. Guo JW, Zhang XT, Chen XS, Zhang XC, Zheng GJ, Zhang BP, et al. Leptomeningeal carcinomatosis as the initial manifestation of gastric adenocarcinoma: A case report. World J Gastroenterol 2014;20:2120-6.

4. Savage NM, Alleyne CH, Vender JR, Figueroa R, Zhang H,
Samuel TA, et al. Dural-based metastatic carcinomas mimicking primary CNS neoplasia: Report of 7 cases emphasizing the role of timely surgery and accurate pathologic evaluation. Int J Clin Exp Pathol 2011;4:530-40.

5. Johnson MD, Powell SZ, Boyer PJ, Well RJ, Moots PL. Dural lesions mimicking meningioma. Hum Pathol 2002;33:1211-26.

How to cite this article: Kapoor A, Savardekar A, Chaterjee D, Salunke P, Vasishta RK, Bhattacharya A. Dural metastasis masquerading as an en plaque meningioma. J Neurosci Rural Pract 2015;6:420-2.

Source of Support: Nil. Conflict of Interest: None declared. 